\title{
Presentación inusual de paniculitis mesentérica
}

\author{
S. RUIZ GARCÍA, M. YEDRA ALCARAZ', N. ORTEGA, M. A. VILLARINOS VIVAS \\ Servicio de Medicina Interna . Hospital Virgen de la Arrixaca. ${ }^{\prime}$ Centro de Salud Barrio \\ del Carmen. Murcia
}

INUSUAL PRESENTATION OF MESENTERIC PANNICULITIS

\begin{abstract}
RESUMEN
La paniculitis mesentérica es un proceso inflamatorio crónico, no neoplásico, que afecta al tejido adiposo del mesenterio fundamentalmente. Están descritas varias formas de presentación clínica, desde asintomática hasta dolor abdominal, afectación del estado general y pérdida de peso. Es excepcional que debute como fiebre aislada. En el tratamiento se ha empleado colchicina, dapsona o corticoides asociados o no a inmunosupresores, pero no hay estudios controlados prospectivos que definan el tratamiento adecuado. Además hay casos de regresión sin terapéutica específica.

A continuación presentamos el caso de un paciente afecto de paniculitis mesentérica con fiebre de predominio vespertino como única manifestación clínica. El tratamiento médico con corticoides orales, durante 2 años, consiguió la remisión clínica de la enfermedad.
\end{abstract}

PALABRAS CLAVE: Paniculitis mesentérica. Mesenteritis retráctil. Fiebre de origen desconocido.

\begin{abstract}
Mesenteric Panniculitis is an inflammatory process, not tumoral, of the adipose tissue of the mesentery. There are documented several ways of clinical presentation, from asymptomatic until abdominal pain, affectation of general condition and loose weight. Excepcionally it is declared like only fever. Several treatments have been used, including colchicine, dapsone or corticosteroids associated or not with inmunosupresants, but there are no prospective controlled studies to define appropiate treatment; moreover, there are cases of regresion without specific therapy.

Below we present the case of a patient affected for mesenteric panniculitis which the one clinical manifestation is fever predominance evening. The treatment with oral corticosteroids, for 2 years, resulted in the disappearance of the clinica.
\end{abstract}

KEY WORDS: Mesenteric paniculitis. Mesenteritis retráctil. Fiebre de origen desconocido.

Ruiz García S, Yedra Alcaraz, M, Ortega N, Villarinos Vivas MA. Presentación inusual de paniculitis mesentérica. An Med Interna (Madrid) 2007; 24: 393-395.

\section{INTRODUCCIÓN}

La paniculitis mesentérica es una enfermedad de causa desconocida y presentación poco frecuente, caracterizada por una extensa inflamación crónica inespecífica que afecta al tejido adiposo del mesenterio de intestino delgado (1). Aparece a partir de la $5^{\text {a }}$ década de la vida y es más frecuente en el sexo masculino. Su diagnóstico es siempre histológico: macrófagos espumosos cargados de lípidos, asociados a fibrosis leve-moderada. Están descritas varias formas clínicas de presentación, desde asintomática $(40 \%)$ hasta dolor abdominal, acompañado de náuseas, fiebre, afectación del estado general, pérdida de peso y masa abdominal palpable (2).

Presentamos a continuación el caso clínico de un paciente con paniculitis mesentérica, con afectación yeyunal, cuya forma de presentación fue fiebre sin foco.

\section{CASO APORTADO}

Varón de 55 años, sin antecedentes de interés, que consulta por fiebre en picos vespertinos de más de 15 días de evolución, con sudoración y mal estado general, sin otros síntomas acompañantes. $\mathrm{La}$ exploración física no revela datos de interés.

Datos de laboratorio: destacan aumento de reactantes de fase aguda (VSG 124, PCR 2,8, fibrinógeno 754) con anemia normocítica normocrómica (Hb 10.6, VCM 79,3, HCM 26,7). Coombs directo negativo. Frotis de sangre periférica normal y sin parásitos intracelulares. Restos de parámetros bioquímicos, incluyendo proteinograma, función tiroidea y orina elemental sin alteraciones. Los hemocultivos y urocultivos fueron repetidamente negativos. Los marcadores tumorales, estudios inmunológicos, así como la serología de virus (VEB, VHC, VHB, VIH), toxoplasma, salmone1la, brucella, lues, Rickettsia y Leishmania, fueron negativos. Mantoux negativo. Exploraciones complementarias: se realiza

Trabajo aceptado: 28 de marzo de 2007 
radiografía tórax, TAC craneal y ecografía abdominal que resultaron normales. Ecocardiografía: válvulas de morfología normal sin flujos patológicos y sin evidencia de vegetaciones. FEVI 65\%. En el TAC abdominal se visualiza imagen de pseudomasa de $6 \times 7 \mathrm{~cm}$ en mesenterio, a nivel de la región de la arteria mesentérica superior e inferior con alternancia de densitometría de partes blandas, con microadenopatías, compatible con paniculitis mesentérica, no pudiendo descartar linfoma, carcinoma, tumor desmoide o carcinoide (Fig. 1).

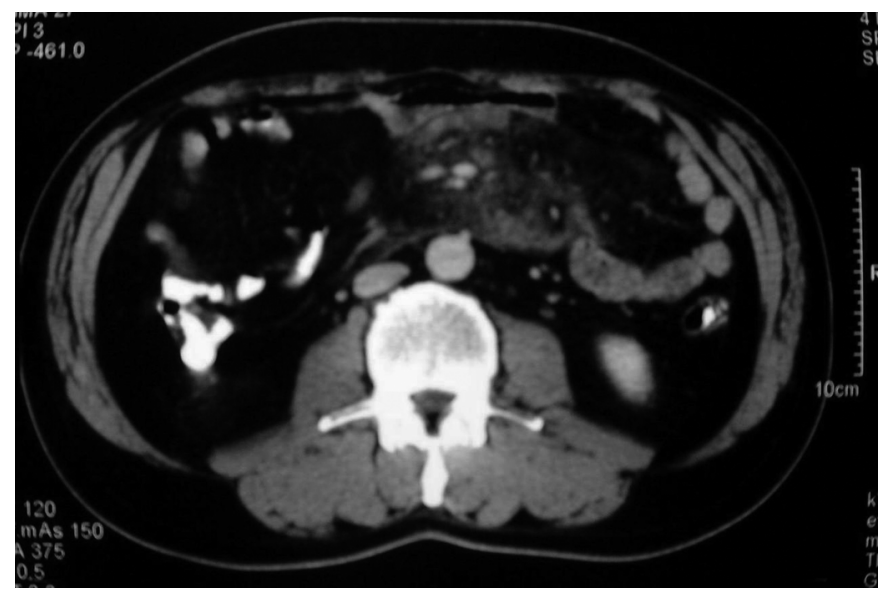

Fig. 1. TAC abdominal que muestra la masa mesentérica anterior a la aorta, en la región media.

Tras los hallazgos de las exploraciones complementarias se intentó tomar biopsia de la masa guiada por TAC, en dos ocasiones, que resultan infructuosas al obtenerse únicamente material necrótico. Se decidió realizar biopsia intraoperatoria de masa abdominal. El informe preliminar habló de esteatonecrosis sin poder descartar malignidad por lo que se amplía la incisión hasta laparotomía media supraumbilical confirmándose la masa en raíz de mesenterio a nivel de primeras asas yeyunales, y se resecan 3-4 cm más.

El informe definitivo de anatomía patológica confirma la sospecha inicial de paniculitis mesentérica. Se trata de grandes extensiones de necrosis grasas con acúmulo de numerosas células vacuoladas de citoplasma espumoso, a veces en torno a pseudoquistes grasos. Se acompaña de infiltrado inflamatorio inespecífico y de fibrosis en las zonas periféricas. Morfológicamente no se observa tumoración subyacente y las células proliferantes se tiñen positivamente para CD68 , lo cual confirma su naturaleza histiocitaria. (Fig. 2).

Tras la cirugía, se inicia tratamiento con corticoides a dosis de 1 $\mathrm{mg} / \mathrm{kg} /$ día, con mejoría del estado general, desapareciendo la fiebre y permaneciendo asintomático durante las revisiones posteriores.

\section{DISCUSIÓN}

La paniculitis mesentérica fue descrita por primera vez por Jura en 1924 como mesenteritis esclerosante. La etiología es muy variada, traumatismos, fármacos, autoinmune, infecciosa, cirugía previa, idiopática y vascular. Tiene buen pronóstico. Clasificada según la Clínica Mayo en:

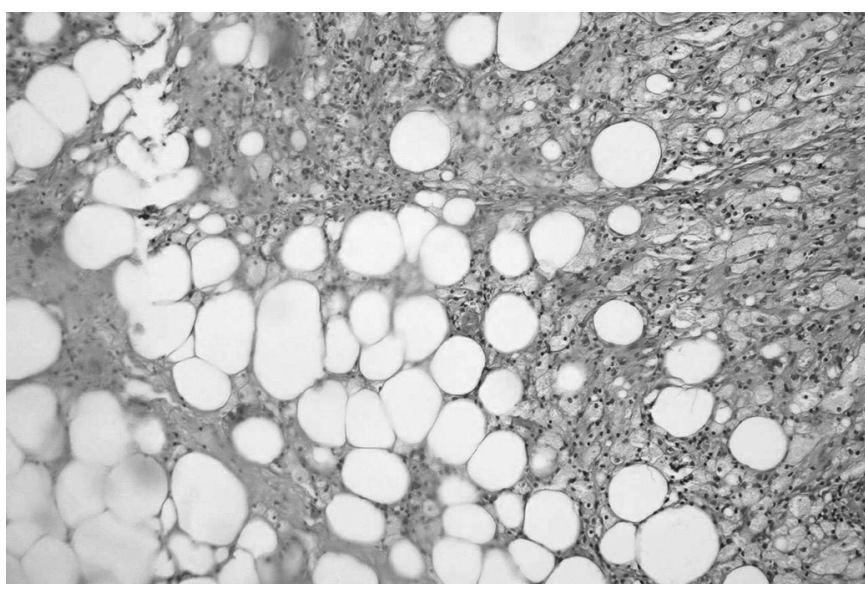

Fig. 2. Imagen microscópica de la lesión donde se observan abundantes adipocitos y sus vacuolas grasas, con un pequeño número de células inflamatorias.

tipo I (42\%), engrosamiento difuso del mesenterio desde raíz a los bordes del intestino delgado; tipo II (32\%), masa nodular aislada en raíz mesentérica; tipo III (20\%), el mesenterio contiene múltiples nódulos de tamaño variable (3). La radiología simple no suele aportar datos, y en el estudio de tránsito baritado y enema opaco, el desplazamiento de asas por un efecto masa es el hallazgo más frecuente (4). En la TAC pueden observarse una o varias masas heterogéneas localizadas en el mesenterio, con densidad diferente según el estadio de la enfermedad y el predominio de inflamación, fibrosis o grasa. El método diagnóstico definitivo es la biopsia de la grasa mesentérica, que se caracteriza por una infiltración variable de macrófagos cargados de grasa asociados a un grado variable de inflamación y fibrosis (5). El diagnóstico diferencial, incluye fibromatosis mesentérica, fibrosarcoma inflamatorio, fibrosis retroperitoneal, pseudotumor inflamatorio, enfermedad de Whipple, liposarcoma inflamatorio, linfoma esclerosante, enfermedad de Weber-Christian y carcinoma metastático ya que pueden dar imágenes radiológicas similares (6). No existe tratamiento específico, en la literatura revisada se han descrito casos de respuesta a esteroides, inmunosupresores, dapsona, colchicina, antibióticos y radioterapia $(3,7-9)$. El tratamiento quirúrgico queda reservado para los casos de obstrucción intestinal extrínseca. En nuestro caso dado que con la biopsia intraoperatoria no se pudo descartar la presencia de tumoración subyacente, se decidió realizar exéresis de la masa abdominal. Posteriormente se instauró tratamiento esteroideo vía oral ante la persistencia de febrícula, quedando el paciente asintomático.

Es destacable en nuestro caso la forma de presentación con fiebre sin otra sintomatología asociada. En la revisión bibliográfica realizada sólo 3 casos debutaron de esta manera (10-12). El resto de casos se presentó como fiebre asociada a dolor abdominal, malestar general y náuseas (13-16). 


\section{Bibliografía}

1. Bari V, Mosharraf SM, Ashraf K, Rafique MZ. Mesenteric paniculitis J Coll Physicians Surg Pak 2004; 14: 117-8

2. Biscaldi E, Romairone E, Rollandi GA. Regarding six cases of mesenteric paniculitis: US, spiral ct, RNM. Radiol Med 2002; 103: 511-8.

3. E. Colomer Rubio, A. Blanes Gallego, C. Carbonell Biot, A. Villar Grimalt, H. Tomas Ivorra, A. Llamusí Llorente. Paniculitis Mesentérica con afectación retroperitoneal resuelta tras tratamiento con pulsos de ciclofosfamida endovenosa. An Med Interna (Madrid) 2003; 20: 31-33.

4. White B, Kong A, Chang AL. Sclerosing mesenteritis. Australas Radiolo 2005; 49: 185-8.

5. Alonso EM, Conde EF, Zameza JJ. Paniculitis mesentérica. Correlación radio-patológica. Seminario - casos - 054. 2001.

6. Azzam I, Croitoru S, Naschitz JE. Sclerosing mesenteritis: a diagnostic challenge. Isr Med Assoc J 2004; 6: 567-8.

7. Miyake H, Sano T, Kamiya J, Nagino M, Uesaka K, Yuasa N, Oda K, Nimuria Y. Successful steroid therapy for postoperative mesenteric panniculitis. Surgery 2003; 133: 118-9.

8. Koornstra JJ, van Olffen GH, van Noort G. Retractile mesenteritis: to treta or not to treat. Hepatogastroenterology 1997; 44: 408-10.

9. Genereau T, Bellin MF, Wechsler B, Le TH, Bellanger J, Grellet J, Godeau P. Demonstration of efficacy of combinig corticoesteroids and colchicine in two patients with idiopathic sclerosing mesenteritis. Dig
Dis Sci 1996; 41: 684-8.

10. Papadaki HA, Kouroumalis EA, Stefanaki K, Roussomoustakaki M, Daskalogiannaki ME, Reppa D, Eliopoulos GD. Retractile mesenteritis presenting as fever of unknown origin and autoinmune haemolytic anaemia. Digestion 2000; 61: 145-8.

11. Hemaidan A, Vanegas F, Alvarez OA, Arroyo MA, Lee M. Mesenteric lipodystropy with fever of unknown origin and mesenteric calcifications. South Med J 1999; 92: 513-6.

12. Sans M, Varas M, Anglada A, Esperanza Bachs M, Navarro S, Brugues J. Mesenteric panniculitis presenting as a fever of unknown origin. Am J Gastroenterol 1995; 90: 1159-61.

13. Parra-Dávila E, Mc Kenney MG, Sleeman D, Hartmann R, Rao RR, McKenney K, et al. Mesenteric Panniculitis: Case report and literature teview. Am Surg 1998; 64: 768-71.

14. Biscaldi E; Romairone E; Rollandi GA. Regarding six cases of mesenteric paniculitis: US, spiral ct, RNM. Radiol Med 2002; 103: 511-8

15. Martínez Odriozola P, García Jiménez N, Cabeza García S, Oceja Barrutieta E. Sclerosing mesenteritis. Report of two cases with different clinical presentation. An Med Interna (Madrid) 2003; 20: 254-6.

16. Hirono S, Sakaguchi S, Iwakura S, Masaki K, Tsuhada K, Yamaue H. Idiopathic isolated omental panniculitis. J Clin Gastroenterol 2005; 39: $79-80$ 